\title{
RESEARCH ON DEVELOPMENT OF COLOR SORTER USING TRIZ
}

\author{
Lihong He, Zhanwen Niu, Dongliang Chen \\ School of Mechanical Engineering, Tianjin University, 300072, China. \\ Email:lihong-he@126.com
}

Abstract: Color Sorter has been widely used in crops further processing industries. And it has a broad market in China. Improving product quality or developing new products is very important for enterprises to keep technical advantages in market competitions. Technology evolution theory can forecast the future technology in products. It can guide a designer along the right direction of developing a new product. The solutions from contradictions solving give starting points to solve a problem. According to these solutions, the designer can detail the design using professional knowledge. This paper is organized as follows. Firstly, it proposes to integrate technology evolution and the design of Color Sorter and forecast the future technologies of the Color Sorter parts, such as the optical signal-collecting system and grooves. Secondly, using the contradiction and inventive principle, this paper proposes several solutions for improving the adjust method of background board.

Key words: Color Sorter, Technology evolution, TRIZ, Contradiction, Inventive principle

\section{INTRODUCTION}

Color Sorter ${ }^{1-3}$ is further processing equipment which can separate materials based on the color aberration between falling particles and background. As is shown in Figure.1, particles are sliding down through grooves to working area from feeder. Two sets of lamps, high sensitive photoelectric detectors, and background boards are fixed on the front and back of the working area. When all particles pass through the work area, photoelectric detectors compare the detected reflex and transmission

Please use the following format when citing this chapter:

He, Lihong, Niu, Zhanwen, Chen, Dongliang, 2006, in International Federation for Information Processing (IFIP), Volume 207, Knowledge Enterprise: Intelligent Strategies In Product Design, Manufacturing, and Management, eds. K. Wang, Kovacs G., Wozny M., Fang M., (Boston: Springer), pp. 465-470. 
luminous flux with benchmark, and then identify those that have different colors. The signal of color aberration is magnified to drive the aerodynamic ejector, which blows away the rejected particles.

Technology is always in evolution. TRIZ ${ }^{4,5}$ was developed in Russia by Genrich Altshuller and his followers in 1940s. It includes many solution tools, such as technology evolution, contradiction, effect and $\mathrm{ARIZ}^{6}$, etc. The technology evolution is very helpful for technology forecasting since they can identify the most promising directions in the development of a system $^{7,8}$.
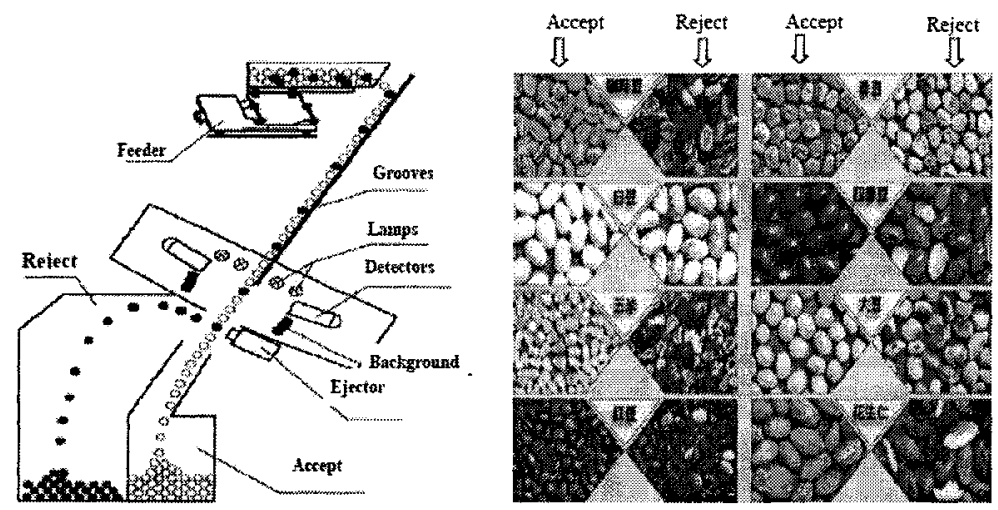

Figure 1. Color Sorter work principle and color sorting efficiency

An inventive situation is usually inherent in the technical and/or physical contradictions in the technique. The technical contradiction represents the confliction between two subsystems of a system. It is the main contradiction in the product evolution. In TRIZ, 39 features ${ }^{9}$ can be used to describe the technical contradictions and 40 inventive principles ${ }^{10}$ are given to address these contradictions.

2.
ANALYSIS OF COLOR SORTER DEVELOPMENT

\subsection{Technology Evolution Analysis and Forecast of Optical Signal Collecting System}

The optical signal-collecting system plays the most important role in color sorter. Its upgrade means the upgrade of the color sorter. The first color 
sorter was made in Britain in 1960s, and now such equipment has evolved into its fourth generation. Based on the evolution laws and principles of TRIZ, the evolution analysis was performed, and the fifth generation was predicted, as is shown in Figure. 2.

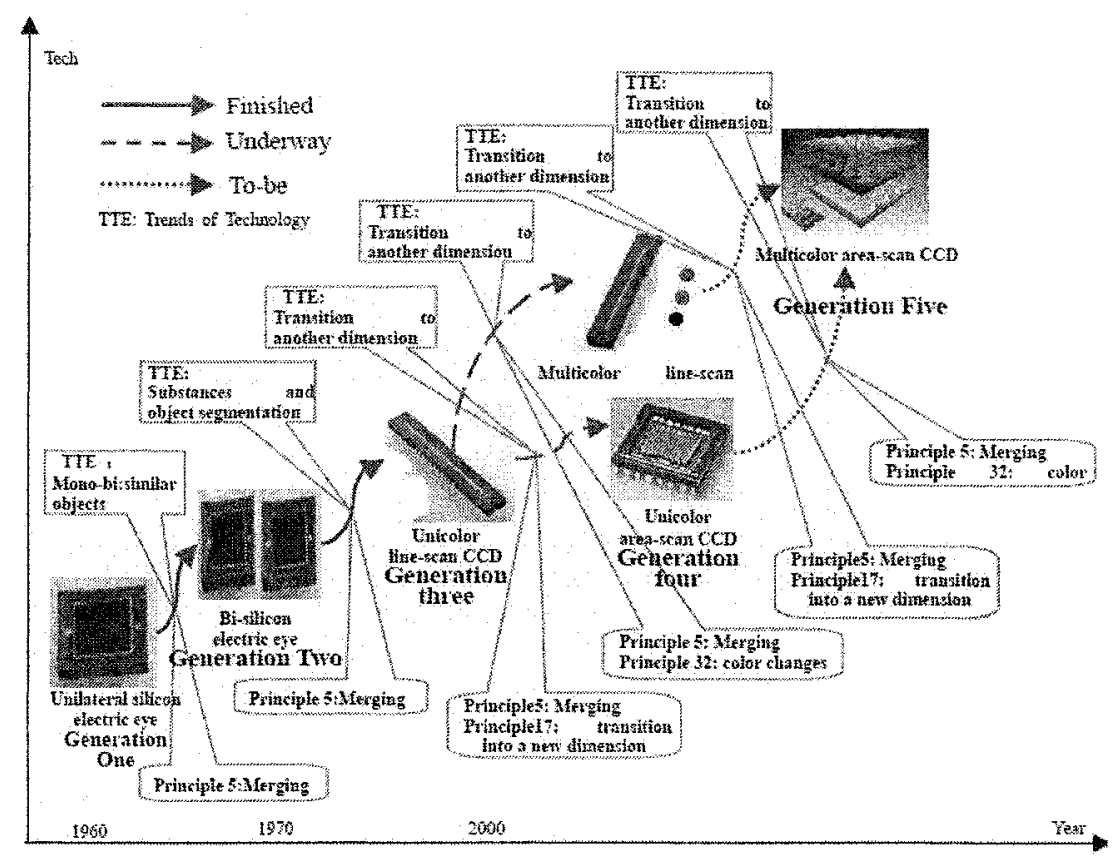

Figure 2. Evolution of optical signal collecting system

Because the unilateral silicon electric eyes that were fixed on the first generation could only catch the unilateral information, color-sorting precision was not enough. According to the laws of increasing flexibility, the eyes were fixed on the front and back of the particles flow in the second generation. More information could be got. The precision of color-sorting was improved. The silicon electric eyes could hardly detect tiny differences because of their large ray-receiving area and low sensitivity. So the area should be subdivided into smaller ones to increase the sensitivity. Based on the law of transition to micro-level, a new photosensitive component called CCD (Charge Coupled Device) was introduced to its' third generation. The unicolor line-scan Camera was first put into use, it worked as many mini eyes worked together. The information collected by CCD system was one hundred times more than before. However, a higher information processing speed was required because of more information to be processed. According to the principle of transition into a new dimension, there were two evolution 
directions in which the fourth generation, one was multicolor line-scan Camera; the other was unicolor area-scan Camera. Unicolor CCD could only perform gray processing while missing large amount of valuable information In addition; the line-scan camera had a poor function of processing multidimension signals. Multicolor line-scan camera based on the RGB-mixture principles could collect all the valuable information contained in the visible light. That broadened the processed spectrum. The application of multicolor line-scan camera and the unicolor area-scan camera not only improved the color-sorting precision, but also broadened the particle sorts.

So, the optical signal collecting system is a processor aiming at getting as more valuable information as possible given that the information processing speed is high enough. According to the laws and principles of TRIZ, we predict that multicolor CCD would be used in the fifth generation of the color sorter.

\subsection{Technology Evolution Forecast of the Grooves}

Corresponding to the optical signal collecting system, grooves were used in color sorter as the main part of particles transmitting system. But it made the area between every two grooves bordered on unoccupied, then the work area utilization ratio was reduced. After the application of CCD technique, the planar signals could be processed; grooves could be replaced by plate. Here we introduced the law of transition to micro-level to separate the relative mass material flow into smaller ones. The work area utilization ratio was enhanced greatly, and the work efficiency of a single Color Sorter

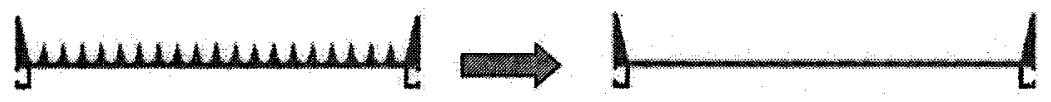

Figure 3. Evolution of the grooves

would also be improved. When using plate, signal interference caused by particle overlap was prevented. Figure 3 shows the improved design of grooves.

\subsection{Improvement of the background board adjust method using contradictions}

For the most popular particles were milk-white rice, the background board was generally milk-white, and it was able to reflect the beam from lamps to the optical signal collecting system, at the same time, the standard 
background lightness could be controlled by changing the rotating the reflection angle of the board. As we know, when specific particles were passing, there must be optimal standard background lightness. With this lightness, the color-sorting precision was the highest theoretically.

The evolution of adjusting method has been evolving through three phases, manual adjust, ordinary motors adjust with feedback, and servomotors adjust, see figure 4 .The servomotor has been widely used in the background lightness adjustment till now.

- Problem description: aiming at improving the applicability but the driver complication must be enhanced.

- Describing the problem with Contradiction matrix is equivalent to:

How to improve the applicability without enhancing the driver complication?

- The Contradiction Matrix suggests the following Inventive Principles:

15-Dynamics (A. Change the object (or outside environment) for optimal performance at every stage of operation. B. Divide an object into parts capable of movement relative to each other. $C$. Change an object from immobile to mobile or adaptive)

29-Pneumatics and hydraulics (A. Use gas and liquid parts of an object instead of solid parts, e.g. inflatable, filled with liquids, air cushion, hydrostatic, hydro-reactive)

37-Thermal expansion (A. Use thermal expansion, or contraction, or materials. B. Use multiple materials with different coefficients of thermal expansion)

28-Mechanics substitution (A. Replace a mechanical means with a sensory (optical, acoustic, taste or smell) means. B. Use electric, magnetic and electromagnetic fields to interact with the object, etc.)

Principle 15 suggested dividing the system into movable parts, the rotating angle fixed to the optimal and obtaining standard lightness by changing the movable background board.

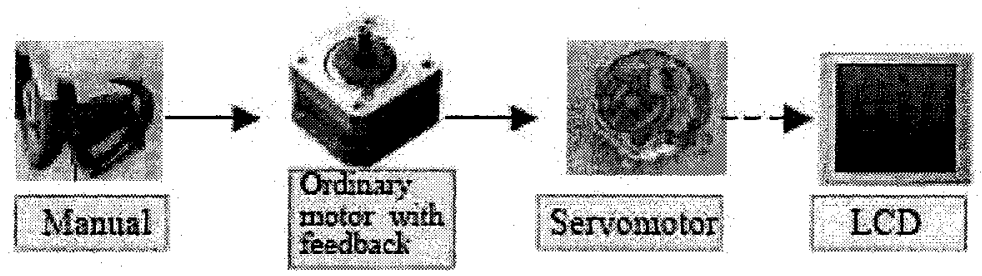

Figure 4. Improvement of the background board adjust method

Principle 28 recommended a starting point to solving the problem, we can replace mechanics with electric. We replaced mechanics with LCD 
lightness adjust system.

Though there were many advantages in servomotor adjust, the ultimate adjusting action was finished by mechanical movement, which was constrained inevitably by machining, material, and the installation precision. With LCD, we could get variable lightness by adjusting input voltage. It would be a breakthrough for the development of the background lightness adjust.

\section{CONCLUSION}

Through studying Color Sorter, using the patterns and principles of technology evolution, the future technologies of the optical signals collecting system and the grooves were given. The new states were the sticking points of new products. Then using the contradiction and inventive principle, this paper proposed several solutions for improving the color-sorting precision.

TRIZ has proved to be a very strong tool in helping to solve difficult technical problems that required inventive thinking. A competitive company pays much attention to the development of new products. Currently, there has been no structured methodology to perform this crucial activity. TRIZ provides a powerful structured methodology to achieve improved products.

\section{REFERENCES}

1.M.C.Pasikatan, F.E.Dowell, High-Speed NIR Segregation of High-and Low-Protein Single Wheat Seeds, Cereal Chem. Vol.81, No.1, 2004, pp.145-150

2.Tom Pearson, Machine Visio System for Automated Detection of Stained Pistachio Nuts, Lebensm. -Wiss.u. -Technolo., 29, 1996,pp.203-209

3.Melchor C.Pasikatan, Floyd E.Dowell, Evaluation of a High-Speed Color Sorter for Segregation of Red and White Wheat; written for presentation at the 2002 ASAE Annual International Meeting/CIGR XVth World Congress, Chicago Illinois, USA, July28-July31, 2002

4.Tan Runhua, The Theory of Inventive Problems Solving, (China Machine PRESS, 2002)

5.Niu Zhanwen, Xu Yanshen, Lin Yue, The Scientific Methodology-TRIZ. China Mechanical Engineering, vol. 10 (1), 1999. pp: 84-89

6.Altshuller G. The Innovation Algorithm, TRIZ, Systematic Innovation and Technical Creativity. Technical Innovation Center, INC., Worcester, 1999

7. Petrov V.M. \& Zlotina E.S., Laws of System Evolution, (Leningrad). 1990

8. Zhang Qinghua, Researching on the Theory of Technology Evolution based on TRIZ and the Engineering Application, MS thesis, Hebei university of technology, 2003

9. Domb Ellen, The 39 Features of Altshuller's Contradiction Matrix(1998); Http: // www.triz-journal.com

10.Tate Karen, Domb Ellen, 40 Principles with Examples(1997); Http: //www.trizjournal.com 